\title{
Bilinear Calderón-Zygmund operators on Sobolev, BMO and Lipschitz spaces
}

Dinghuai Wang and Jiang Zhou*

${ }^{\text {*Correspondence: }}$

zhoujiangshuxue@126.com

College of Mathematics and System

Sciences, Xinjiang University,

Urumqi, 830046, Republic of China

\begin{abstract}
In this paper, the authors establish the necessary and sufficient condition such that the bilinear Calderón-Zygmund operators are bounded from $L_{i p_{\alpha}}\left(\mathbb{R}^{n}\right) \times L^{n / \alpha}\left(\mathbb{R}^{n}\right)$ to $B M O\left(\mathbb{R}^{n}\right)$ space and from $L i p_{\alpha}\left(\mathbb{R}^{n}\right) \times L^{p}\left(\mathbb{R}^{n}\right)$ to $L i p_{\alpha-n / p}\left(\mathbb{R}^{n}\right)$ space. As an application, the bilinear Riesz transform is a good example which meets the related conditions.

Furthermore, the authors also establish another necessary and sufficient condition for the bilinear Calderón-Zygmund operators to be bounded from $L i p_{\alpha}\left(\mathbb{R}^{n}\right) \times B M O\left(\mathbb{R}^{n}\right)$ to $L i p_{\alpha}\left(\mathbb{R}^{n}\right)$ space, from $L i p_{\alpha_{1}}\left(\mathbb{R}^{n}\right) \times L i p_{\alpha_{2}}\left(\mathbb{R}^{n}\right)$ to $L i p_{\alpha_{1}+\alpha_{2}}\left(\mathbb{R}^{n}\right)$ space, and from $L i p_{\alpha}\left(\mathbb{R}^{n}\right) \times \dot{B}^{s}\left(\mathbb{R}^{n}\right)$ to $\dot{B}^{s-\alpha}\left(\mathbb{R}^{n}\right)$ space.
\end{abstract}

MSC: 42B20; 42B35; 47B38

Keywords: BMO space; commutator; Lipschitz space; Morrey space

\section{Introduction}

It is well known that standard singular integral theory has been going on for a long period and plays an important role in the study of the classical harmonic analysis. Many authors have considered the boundedness of generalized singular integrals (non-convolution operators)

$$
T f(x)=\int_{\mathbb{R}^{n}} K(x, y) f(y) d y,
$$

on several function spaces (see [1-6]). In recent years, multilinear singular integrals of Calderón-Zygmund type and fractional integral type have attracted a lot of attention (see [7-14]). Many results which parallel the linear theory of classical Calderón-Zygmund operators are obtained. The study of multilinear operators is not motivated by a mere quest to generalize the theory of linear operators but rather by their natural appearance in analysis.

Let $T$ be a multilinear operator initially defined on the $m$-fold product of Schwartz spaces and taking values in the space of tempered distributions,

$$
T: S\left(\mathbb{R}^{n}\right) \times \cdots \times S\left(\mathbb{R}^{n}\right) \rightarrow S^{\prime}\left(\mathbb{R}^{n}\right)
$$

The $m$-linear Calderón-Zygmund operators $T$ is defined by

$$
T\left(f_{1}, \ldots, f_{m}\right)(x)=\int_{\left(\mathbb{R}^{n}\right)^{m}} K\left(x, y_{1}, \ldots, y_{m}\right) f_{1}\left(y_{1}\right) \cdots f_{m}\left(y_{m}\right) d y_{1} \cdots d y_{m},
$$

(c) 2015 Wang and Zhou. This article is distributed under the terms of the Creative Commons Attribution 4.0 International License (http://creativecommons.org/licenses/by/4.0/), which permits unrestricted use, distribution, and reproduction in any medium, provided you give appropriate credit to the original author(s) and the source, provide a link to the Creative Commons license, and indicate if changes were made. 
where $K\left(x, y_{1}, \ldots, y_{m}\right)$ is a locally integrable function defined away from the diagonal $x=$ $y_{1}=\cdots=y_{m}$ in $\left(\mathbb{R}^{n}\right)^{m+1}$, and it satisfies the following estimates:

$$
\begin{aligned}
& \left|K\left(x, y_{1}, \ldots, y_{m}\right)\right| \leq \frac{A}{\left(\left|x-y_{1}\right|+\cdots+\left|x-y_{m}\right|\right)^{m n}}, \\
& \left|K\left(x, y_{1}, \ldots, y_{j}, \ldots, y_{m}\right)-K\left(x^{\prime}, y_{1}, \ldots, y_{j}, \ldots, y_{m}\right)\right| \\
& \quad \leq \frac{A\left|x-x^{\prime}\right|^{\delta}}{\left(\left|x-y_{1}\right|+\cdots+\left|x-y_{m}\right|\right)^{m n+\delta}}
\end{aligned}
$$

whenever $\left|x-x^{\prime}\right| \leq \frac{1}{2} \max _{1 \leq j \leq m}\left|x-y_{j}\right|$ and also for each $j$

$$
\begin{aligned}
& \left|K\left(x, y_{1}, \ldots, y_{j}, \ldots, y_{m}\right)-K\left(x, y_{1}, \ldots, y_{j}^{\prime}, \ldots, y_{m}\right)\right| \\
& \quad \leq \frac{A\left|y_{j}-y_{j}^{\prime}\right|^{\delta}}{\left(\left|x-y_{1}\right|+\cdots+\left|x-y_{m}\right|\right)^{m n+\delta}}
\end{aligned}
$$

whenever $\left|y_{j}-y_{j}^{\prime}\right| \leq \frac{1}{2} \max _{1 \leq j \leq m}\left|x-y_{j}\right|$. The class of all functions satisfying (1.1), (1.2), and (1.3) with parameters $m$ and $\delta$ will be denoted by $m-\mathrm{CZK}(A, \delta)$. The $m$-linear Calder'onZygmund operator $T$ with $m$ - $\mathrm{CZK}(A, \delta)$ will be denoted by $m-\mathrm{CZO}(A, \delta)$.

In 2001, Grafakos and Torres in [10] have obtained the boundedness properties of operators $T$ with kernels $K$ in the class $m$ - $\operatorname{CZK}(A, \delta)$ from a product of $L^{p}$ spaces into another Lebesgue space, endpoint weak type estimates, interpolation, a multilinear $T 1$ theorem and a variety of results regarding multilinear multiplier operators. In [11], it was proved that $T$ is bounded from $H^{p_{1}} \times \cdots \times H^{p_{m}}$ to $L^{p}$ with $\frac{1}{p}=\sum_{j=1}^{m} \frac{1}{p_{j}}$ by showing that $\left\|T\left(a_{1}, \ldots, a_{m}\right)\right\|_{L^{p}} \leq C$, where $a_{j}$ are $H^{p_{j}}$-atoms. However, some other classical function spaces are not still considered for these operators with $m \geq 2$, although many remarkable results have been established for them in the case $m=1$; see $[2,3,5,6]$.

Next, we define a class of singular integrals which we will consider in this paper, and we establish the necessary and sufficient condition such that this operators are bounded on BMO, Lipschitz, and Sobolev spaces. As an application, the bilinear Riesz transform in the first variable is a good example which meets the related conditions.

\section{Definitions and main results}

We first give the definition of bilinear Calderón-Zygmund operators.

Definition 2.1 Let $0<\alpha<\delta \leq 1$, for $f_{1} \in \operatorname{Lip}\left(\mathbb{R}_{\alpha}\right)$ and the bounded function $f_{2}$, we define

$$
\tilde{T}_{\epsilon}\left(f_{1}, f_{2}\right)(x)=\int_{\mathbb{R}^{n}} \int_{\mathbb{R}^{n}}\left(K_{\epsilon}\left(x, y_{1}, y_{2}\right)-K_{1}\left(x_{0}, y_{1}, y_{2}\right)\right) f_{1}\left(y_{1}\right) f_{2}\left(y_{2}\right) d y_{1} d y_{2},
$$

where $x_{0}$ is a fixed point in $\mathbb{R}^{n}$, the singular kernels $K\left(x, y_{1}, y_{2}\right)$ satisfying (1.1), (1.2), (1.3),

$$
\lim _{\epsilon \rightarrow 0} \int_{\epsilon<\left|x-y_{1}\right|<1} K_{f_{2}}\left(x, y_{1}\right) d y_{1} \text { exists for almost every point } x,
$$

and for any $0<r<R$,

$$
\left|\int_{r<\left|x-y_{1}\right|<R} \int_{r<\left|x-y_{2}\right|<R} K\left(x, y_{1}, y_{2}\right) d y_{1} d y_{2}\right| \leq C,
$$


here

$$
K_{\epsilon}\left(x, y_{1}, y_{2}\right)=K\left(x, y_{1}, y_{2}\right) \chi_{\left\{\left|x-y_{1}\right|>\epsilon\right\}}\left(y_{1}\right) \chi_{\left\{\left|x-y_{2}\right|>\epsilon\right\}}\left(y_{2}\right)
$$

and

$$
K_{f_{2}}\left(x, y_{1}\right)=\int_{\mathbb{R}^{n}} K\left(x, y_{1}, y_{2}\right) f_{2}\left(y_{2}\right) d y_{2} .
$$

Then we also define

$$
\tilde{T}\left(f_{1}, f_{2}\right)(x)=\lim _{\epsilon \rightarrow 0} \tilde{T}_{\epsilon}\left(f_{1}, f_{2}\right)(x) .
$$

Remark 2.1 (i) By [10], Lemma 2, and $f_{2} \in L^{\infty}\left(\mathbb{R}^{n}\right)$, we see that $K_{f_{2}}\left(x, y_{1}\right)$ is in 1- $\mathrm{CZK}\left(c_{n}\left\|f_{2}\right\|_{L^{\infty}} A, \epsilon\right)$. Thus, condition (2.2) is also classical for singular integrals of principal value type. Condition (2.3) is a weak cancellation condition that is also present in the linear case (see $[5,15])$.

(ii) It follows from the properties of $K\left(x, y_{1}, y_{2}\right)$ that the limit exists almost everywhere. Indeed,

$$
\begin{aligned}
\tilde{T}_{\epsilon}\left(f_{1}, f_{2}\right)(x)= & \int_{\mathbb{R}^{n}} \int_{\left|x-y_{1}\right|<1} K_{\epsilon}\left(x, y_{1}, y_{2}\right)\left(f_{1}(y)-f_{1}(x)\right) f_{2}\left(y_{2}\right) d y_{1} d y_{2} \\
& +\left(\int_{\mathbb{R}^{n}} \int_{\left|x-y_{1}\right|<1} K_{\epsilon}\left(x, y_{1}, y_{2}\right) d y_{1} f_{2}\left(y_{2}\right) d y_{2}\right) f_{1}(x) \\
& +\int_{\mathbb{R}^{n}} \int_{\mathbb{R}^{n}}\left(K_{1}\left(x, y_{1}, y_{2}\right)-K_{1}\left(x_{0}, y_{1}, y_{2}\right)\right)\left(f_{1}\left(y_{1}\right)-f_{1}(x)\right) f_{2}\left(y_{2}\right) d y_{1} d y_{2} \\
& +\int_{\mathbb{R}^{n}} \int_{\mathbb{R}^{n}}\left(K_{1}\left(x, y_{1}, y_{2}\right)-K_{1}\left(x_{0}, y_{1}, y_{2}\right)\right) f_{2}\left(y_{2}\right) d y_{1} d y_{2} f_{1}(x),
\end{aligned}
$$

where the first, third, and fourth integrals are absolutely convergent. The second integral converges by property (2.2) of kernel $K$.

(iii) If the functions $f_{1}$ and $f_{2}$ have compact support, then the difference between $T$ and $\tilde{T}$ is the constant

$$
-\int_{\mathbb{R}^{n}} \int_{\mathbb{R}^{n}} K_{1}\left(x_{0}, y_{1}, y_{2}\right) f_{1}\left(y_{1}\right) f_{2}\left(y_{2}\right) d y_{1} d y_{2},
$$

this slight discrepancy, however, will cause no ambiguities when $T\left(f_{1}, f_{2}\right)$ is seen as an element of $B M O$ or $\operatorname{Lip}_{\alpha}\left(\mathbb{R}^{n}\right)$.

We recall the definitions of some classical function spaces which will be considered in this paper.

Definition 2.2 The BMO space is defined by

$$
B M O\left(\mathbb{R}^{n}\right)=\left\{f \in L_{\mathrm{loc}}^{1}:\|f\|_{B M O\left(\mathbb{R}^{n}\right)}=\sup _{x \in \mathbb{R}^{n}, r>0} \frac{1}{|B(x, r)|} \int_{B(x, r)}\left|f(y)-m_{B}(f)\right| d y<\infty\right\},
$$

where $m_{B}(f)=\frac{1}{|B|} \int_{B} f(y) d y$. 
Definition 2.3 Let $0<\alpha<1$. The Lipschitz space is defined by

$$
\operatorname{Lip}_{\alpha}\left(\mathbb{R}^{n}\right)=\left\{f:\|f\|_{L i p_{\alpha}\left(\mathbb{R}^{n}\right)}=\sup _{x \neq y} \frac{|f(x)-f(y)|}{|x-y|^{\alpha}}<\infty\right\} .
$$

Remark 2.2 Note that, for $1 \leq p \leq \infty$,

$$
\|f\|_{L i p_{\alpha}\left(\mathbb{R}^{n}\right)} \approx \sup _{x \in \mathbb{R}^{n}, r>0} \frac{1}{|B(x, r)|^{1 / p+\alpha / n}}\left(\int_{B(x, r)}\left|f(y)-m_{B}(f)\right|^{p} d y\right)^{1 / p} .
$$

Thus, for $\alpha=0$, the space $\operatorname{Lip}_{\alpha}\left(\mathbb{R}^{n}\right)$ coincides with the space $B M O\left(\mathbb{R}^{n}\right)$. If we identify two functions whose difference is a constant, then the BMO and the Lipschitz space will become a Banach space.

Definition 2.4 Let $0<s<1$. The homogeneous Sobolev space is defined by

$$
\dot{B}^{s}\left(\mathbb{R}^{n}\right)=\left\{f:\|f\|_{\dot{B}^{s}\left(\mathbb{R}^{n}\right)}=\left(\int_{\mathbb{R}^{n}} \int_{\mathbb{R}^{n}} \frac{|f(x)-f(y)|^{2}}{|x-y|^{n+2 s}} d x d y\right)^{1 / 2}<\infty\right\}
$$

There are two remarkable results on the Lipschitz spaces and the homogeneous Sobolev spaces boundedness of Calderón-Zygmund operators.

Proposition 2.1 ([3]) Let $0<\alpha<\delta \leq 1$. If $T$ is a $\mathrm{CZO}(\delta)$ and $T 1=0$, then $T$ is bounded on $\operatorname{Lip}_{\alpha}\left(\mathbb{R}^{n}\right)$.

Proposition 2.2 ([3]) Let $0<s<\delta \leq 1$. If $T$ is a $\mathrm{CZO}(\delta)$ and $T 1=0$, then $T$ is bounded on $\dot{B}^{s}\left(\mathbb{R}^{n}\right)$.

Motivated by Proposition 2.1 and Proposition 2.2, we obtain the following theorems.

Theorem 2.1 Let $0<\alpha<\delta \leq 1$ and $\tilde{T}$ can be extended to a bounded operator from $L^{p_{1}} \times$ $L^{n / \alpha}$ into $L^{p}$, where $1<p, p_{1}<\infty$ with $1 / p=1 / p_{1}+\alpha / n$. The following two statements are equivalent:

(i) For $f_{1} \in \operatorname{Lip}\left(\mathbb{R}^{n}\right)$ and $f_{2} \in L^{n / \alpha}\left(\mathbb{R}^{n}\right)$, we have

$$
\left\|\tilde{T}\left(f_{1}, f_{2}\right)\right\|_{B M O\left(\mathbb{R}^{n}\right)} \lesssim\left\|f_{1}\right\|_{L i p_{\alpha}\left(\mathbb{R}^{n}\right)}\left\|f_{2}\right\|_{L^{n / \alpha}\left(\mathbb{R}^{n}\right)} .
$$

(ii) $\tilde{T}\left(1, f_{2}\right)(x) \equiv 0$.

Theorem 2.2 Let $0<\alpha<\delta \leq 1, n / \alpha<p \leq \infty$. The following two statements are equivalent:

(i) For $f_{1} \in \operatorname{Lip}\left(\mathbb{R}^{n}\right)$ and $f_{2} \in L^{p}\left(\mathbb{R}^{n}\right)$, we have

$$
\left\|\tilde{T}\left(f_{1}, f_{2}\right)\right\|_{L i p_{\alpha-n / p}\left(\mathbb{R}^{n}\right)} \lesssim\left\|f_{1}\right\|_{L i p_{\alpha}\left(\mathbb{R}^{n}\right)}\left\|f_{2}\right\|_{L^{p}\left(\mathbb{R}^{n}\right)} .
$$

(ii) $\tilde{T}\left(1, f_{2}\right)(x) \equiv 0$. 
Example Let $R_{1}$ be the bilinear Riesz transform in the first variable

$$
R_{1}\left(f_{1}, f_{2}\right)(x)=p \cdot v \cdot \int_{\mathbb{R}} \int_{\mathbb{R}} \frac{x-y_{1}}{\left|\left(x-y_{1}, x-y_{2}\right)\right|^{3}} f_{1}\left(y_{1}\right) f_{2}\left(y_{2}\right) d y_{1} d y_{2} .
$$

It is easy to see that $\tilde{R}_{1}\left(1, f_{2}\right)(x)=0$ and $K\left(x, y_{1}, y_{2}\right)=\frac{x-y_{1}}{\left|\left(x-y_{1}, x-y_{2}\right)\right|^{3}}$ satisfying (1.1), (1.2), (1.3), (2.2), and (2.3), then we have the following results.

Corollary 2.1 Let $0<\alpha<1$. Then $\tilde{R}_{1}$ is a bounded operator from Lip $\operatorname{LR}_{\alpha}\left(\mathbb{R}^{n}\right) \times L^{n / \alpha}\left(\mathbb{R}^{n}\right)$ to $B M O\left(\mathbb{R}^{n}\right)$.

Corollary 2.2 Let $0<\alpha<1$ and $n / \alpha<p \leq \infty$. Then $\tilde{R}_{1}$ is a bounded operator from $\operatorname{Lip}_{\alpha}\left(\mathbb{R}^{n}\right) \times L^{p}\left(\mathbb{R}^{n}\right)$ to $\operatorname{Lip}_{\alpha-n / p}\left(\mathbb{R}^{n}\right)$.

Theorem 2.3 Let $0<\alpha<\delta \leq 1$ and $\tilde{T}$ can be extended to a bounded operator from $L^{p_{1}} \times$ $L^{p_{2}}$ into $L^{p}$, where $1<p, p_{1}, p_{2}<\infty$ with $1 / p=1 / p_{1}+1 / p_{2}$. The following two statements are equivalent:

(i) For $f_{1} \in \operatorname{Lip}\left(\mathbb{R}^{n}\right)$ and $f_{2} \in B M O\left(\mathbb{R}^{n}\right)$, we have

$$
\left\|\tilde{T}\left(f_{1}, f_{2}\right)\right\|_{L i p_{\alpha}\left(\mathbb{R}^{n}\right)} \lesssim\left\|f_{1}\right\|_{L i p_{\alpha}\left(\mathbb{R}^{n}\right)}\left\|f_{2}\right\|_{B M O\left(\mathbb{R}^{n}\right)}
$$

(ii) If there exists some $j \in\{1,2\}$ such that $f_{j}(x) \equiv 1$, then $\tilde{T}\left(f_{1}, f_{2}\right)(x) \equiv 0$.

Theorem 2.4 Let $0<\alpha_{1}, \alpha_{2}<\delta \leq 1$ with $\alpha_{1}+\alpha_{2}<\delta$. The following two statements are equivalent:

(i) For $f_{1} \in \operatorname{Lip}_{\alpha_{1}}\left(\mathbb{R}^{n}\right)$ and $f_{2} \in \operatorname{Lip} \alpha_{\alpha_{2}}\left(\mathbb{R}^{n}\right)$, we have

$$
\left\|\tilde{T}\left(f_{1}, f_{2}\right)\right\|_{\operatorname{Lip}_{\alpha_{1}+\alpha_{2}}\left(\mathbb{R}^{n}\right)} \lesssim\left\|f_{1}\right\|_{\operatorname{Lip}_{\alpha_{1}}\left(\mathbb{R}^{n}\right)}\left\|f_{2}\right\|_{\operatorname{Lip}_{\alpha_{2}}\left(\mathbb{R}^{n}\right)}
$$

(ii) If there exists some $j \in\{1,2\}$ such that $f_{j}(x) \equiv 1$, then $\tilde{T}\left(f_{1}, f_{2}\right)(x) \equiv 0$.

Theorem 2.5 Let $0<\alpha<s<\delta \leq 1$. The following two statements are equivalent:

(i) For $f_{1} \in \operatorname{Lip} \alpha\left(\mathbb{R}^{n}\right)$ and $f_{2} \in \dot{B}^{s}\left(\mathbb{R}^{n}\right)$, we have

$$
\left\|T\left(f_{1}, f_{2}\right)\right\|_{\dot{B}^{s-\alpha}\left(\mathbb{R}^{n}\right)} \lesssim\left\|f_{1}\right\|_{L i p_{\alpha}\left(\mathbb{R}^{n}\right)}\left\|f_{2}\right\|_{\dot{B}^{s}\left(\mathbb{R}^{n}\right)} .
$$

(ii) If there exists some $j \in\{1,2\}$ such that $f_{j}(x) \equiv 1$, then $T\left(f_{1}, f_{2}\right)(x) \equiv 0$.

\section{Proofs of Theorems 2.1-2.5}

For simplicity, we only give the proof of Theorem 2.1, Theorem 2.4, and Theorem 2.5. For the proof of Theorem 2.2 and Theorem 2.3 similar arguments are applied with necessary modifications.

Proof of Theorem 2.1 (ii) $\Rightarrow$ (i). For any $x, y \in Q$, we have

$$
\begin{aligned}
\tilde{T}\left(f_{1}, f_{2}\right)(x)-\tilde{T}\left(f_{1}, f_{2}\right)(y) & =\lim _{\epsilon \rightarrow 0}\left(\tilde{T}_{\epsilon}\left(f_{1}, f_{2}\right)(x)-\tilde{T}_{\epsilon}\left(f_{1}, f_{2}\right)(y)\right) \\
& =\lim _{\epsilon \rightarrow 0} \int_{\mathbb{R}^{n}} \int_{\mathbb{R}^{n}}\left(K_{\epsilon}\left(x, y_{1}, y_{2}\right)-K_{\epsilon}\left(y, y_{1}, y_{2}\right)\right) f_{1}\left(y_{1}\right) f_{2}\left(y_{2}\right) d y_{1} d y_{2} .
\end{aligned}
$$


Then condition (ii) implies that

$$
\lim _{\epsilon \rightarrow 0} \int_{\mathbb{R}^{n}} \int_{\mathbb{R}^{n}}\left(K_{\epsilon}\left(x, y_{1}, y_{2}\right)-K_{\epsilon}\left(y, y_{1}, y_{2}\right)\right) f_{2}\left(y_{2}\right) d y_{1} d y_{2}=0
$$

Therefore,

$$
\begin{aligned}
& \tilde{T}\left(f_{1}, f_{2}\right)(x)-\tilde{T}\left(f_{1}, f_{2}\right)(y) \\
&=\lim _{\epsilon \rightarrow 0} \int_{\mathbb{R}^{n}} \int_{\mathbb{R}^{n}}\left(K_{\epsilon}\left(x, y_{1}, y_{2}\right)-K_{\epsilon}\left(y, y_{1}, y_{2}\right)\right)\left(f_{1}\left(y_{1}\right)-\lambda\right) f_{2}\left(y_{2}\right) d y_{1} d y_{2} \\
&=\lim _{\epsilon \rightarrow 0} \int_{\mathbb{R}^{n}} \int_{\mathbb{R}^{n}} K_{\epsilon}\left(x, y_{1}, y_{2}\right)\left(f_{1}\left(y_{1}\right)-\lambda\right) f_{2}\left(y_{2}\right) \chi_{2 B}\left(y_{1}\right) d y_{1} d y_{2} \\
&-\lim _{\epsilon \rightarrow 0} \int_{\mathbb{R}^{n}} \int_{\mathbb{R}^{n}} K_{\epsilon}\left(y, y_{1}, y_{2}\right)\left(f_{1}\left(y_{1}\right)-\lambda\right) f_{2}\left(y_{2}\right) \chi_{2 B}\left(y_{1}\right) d y_{1} d y_{2} \\
&+\lim _{\epsilon \rightarrow 0} \int_{\mathbb{R}^{n}} \int_{\mathbb{R}^{n}}\left(K_{\epsilon}\left(x, y_{1}, y_{2}\right)-K_{\epsilon}\left(y, y_{1}, y_{2}\right)\right)\left(f_{1}\left(y_{1}\right)-\lambda\right) f_{2}\left(y_{2}\right) \chi_{(2 B) c}\left(y_{1}\right) \chi_{2 B}\left(y_{2}\right) d y_{1} d y_{2} \\
&+\lim _{\epsilon \rightarrow 0} \int_{\mathbb{R}^{n}} \int_{\mathbb{R}^{n}}\left(K_{\epsilon}\left(x, y_{1}, y_{2}\right)-K_{\epsilon}\left(y, y_{1}, y_{2}\right)\right)\left(f_{1}\left(y_{1}\right)-\lambda\right) \\
& \times f_{2}\left(y_{2}\right) \chi_{(2 B)^{c}}\left(y_{1}\right) \chi_{(2 B) c}\left(y_{2}\right) d y_{1} d y_{2} \\
&:= \mathrm{I}_{1}+\mathrm{I}_{2}+\mathrm{I}_{3}+\mathrm{I}_{4},
\end{aligned}
$$

where $B=B(x, r)$ with $r=|x-y|$ taking $\epsilon<r$ and $\lambda=m_{B}\left(f_{1}\right)$.

Note that $T$ is a bounded operator from $L^{p_{1}}\left(\mathbb{R}^{n}\right) \times L^{n / \alpha}\left(\mathbb{R}^{n}\right)$ to $L^{p}\left(\mathbb{R}^{n}\right)$ with $1 / p=1 / p_{1}+$ $\alpha / n$, then

$$
\begin{aligned}
\frac{1}{|Q|} \int_{Q}\left|\mathrm{I}_{1}\right| d x & \lesssim \frac{1}{|Q|} \int_{Q}\left|T\left(\left(f_{1}-\lambda\right) \chi_{2 B}, f_{2}\right)(x)\right| d x \\
& \lesssim\left\{\frac{1}{|Q|} \int_{Q}\left|T\left(\left(f_{1}-\lambda\right) \chi_{2 B}, f_{2}\right)(x)\right|^{p} d x\right\}^{1 / p} \\
& \lesssim \frac{1}{|Q|^{1 / p}}\left\|\left(f_{1}-\lambda\right) \chi_{2 B}\right\|_{L^{p_{1}\left(\mathbb{R}^{n}\right)}}\left\|f_{2}\right\|_{L^{n / \alpha}\left(\mathbb{R}^{n}\right)} \\
& \lesssim \frac{|2 B|^{1 / p}}{|Q|^{1 / p}}\left\|f_{1}\right\|_{L_{i p_{\alpha}}\left(\mathbb{R}^{n}\right)}\left\|f_{2}\right\|_{L^{n / \alpha}\left(\mathbb{R}^{n}\right)} \\
& \lesssim\left\|f_{1}\right\|_{L_{i p_{\alpha}}\left(\mathbb{R}^{n}\right)}\left\|f_{2}\right\|_{L^{n / \alpha}\left(\mathbb{R}^{n}\right)} .
\end{aligned}
$$

Similarly, by $B(x, 2 r) \subset B(y, 3 r)$, we also have

$$
\frac{1}{|Q|} \int_{Q}\left|\mathrm{I}_{2}\right| d y \lesssim\left\|f_{1}\right\|_{L_{i p_{\alpha}}\left(\mathbb{R}^{n}\right)}\left\|f_{2}\right\|_{L^{n / \alpha}\left(\mathbb{R}^{n}\right)}
$$

For $\mathrm{I}_{3}$, it follows from $y_{1} \in(2 B)^{c}$ that $|x-y| \leq 1 / 2\left|x-y_{1}\right|$, and choosing $\beta$ such that $0<\alpha<\beta$, then

$$
\begin{aligned}
\left|\mathrm{I}_{3}\right| & \lesssim \int_{\mathbb{R}^{n}} \int_{\mathbb{R}^{n}} \frac{|x-y|^{\delta}}{\left(\left|x-y_{1}\right|+\left|x-y_{2}\right|\right)^{2 n+\delta}}\left|\left(f_{1}\left(y_{1}\right)-\lambda\right) \chi_{(2 B)^{c}}\left(y_{1}\right)\right|\left|f_{2}\left(y_{2}\right) \chi_{2 B}\left(y_{2}\right)\right| d y_{1} d y_{2} \\
& \lesssim|x-y|^{\delta} \int_{(2 B)^{c}} \frac{\left|f_{1}\left(y_{1}\right)-\lambda\right|}{\left|x-y_{1}\right|^{n+\beta+\delta}} d y_{1} \int_{2 B} \frac{\left|f_{2}\left(y_{2}\right)\right|}{\left|x-y_{2}\right|^{n-\beta}} d y_{2}
\end{aligned}
$$




$$
\begin{aligned}
& \lesssim|x-y|^{\delta+\beta-\alpha}\left\|f_{2}\right\|_{L^{n / \alpha}\left(\mathbb{R}^{n}\right)} \sum_{k=1}^{\infty} \int_{2^{k+1} B \backslash 2^{k} B} \frac{\left|f_{1}\left(y_{1}\right)-\lambda\right|}{\left|x-y_{1}\right|^{n+\beta+\delta}} d y_{1} \\
& \lesssim|x-y|^{\delta+\beta-\alpha}\left\|f_{2}\right\|_{L^{n / \alpha}\left(\mathbb{R}^{n}\right)} \sum_{k=1}^{\infty}(k+1)\left(2^{k}|x-y|\right)^{\alpha-\beta-\delta}\left\|f_{1}\right\|_{L i p_{\alpha}\left(\mathbb{R}^{n}\right)} \\
& \lesssim\left\|f_{1}\right\|_{L i p_{\alpha}\left(\mathbb{R}^{n}\right)}\left\|f_{2}\right\|_{L^{n / \alpha}\left(\mathbb{R}^{n}\right)} .
\end{aligned}
$$

Choosing $0<\beta_{1}<\delta-\alpha$, we have

$$
\begin{aligned}
\left|I_{4}\right| & \lesssim \int_{(2 B)^{c}} \int_{(2 B) c} \frac{|x-y|^{\delta}}{\left(\left|x-y_{1}\right|+\left|x-y_{2}\right|\right)^{2 n+\delta}}\left|\left(f_{1}\left(y_{1}\right)-\lambda\right)\right|\left|f_{2}\left(y_{2}\right)\right| d y_{1} d y_{2} \\
& \lesssim|x-y|^{\delta} \int_{(2 B)^{c}} \frac{\left|f_{1}\left(y_{1}\right)-\lambda\right|}{|x-y|^{n-\beta_{1}+\delta}} d y_{1} \int_{(2 B)^{c}} \frac{\left|f_{2}\left(y_{2}\right)\right|}{\left|x-y_{2}\right|^{n+\beta_{1}}} d y_{2} \\
& \lesssim|x-y|^{\delta-\beta_{1}-\alpha}|| f_{2} \|_{L^{n / \alpha}\left(\mathbb{R}^{n)}\right.} \sum_{k=1}^{\infty} \int_{2^{k+1} B \backslash 2^{k} B} \frac{\left|f_{1}\left(y_{1}\right)-\lambda\right|}{|x-y|^{n-\beta_{1}+\delta}} d y_{1} \\
& \lesssim|x-y|^{\delta-\beta_{1}-\alpha}\left\|f_{2}\right\|_{L^{n / \alpha}\left(\mathbb{R}^{n)}\right.} \sum_{k=1}^{\infty}(k+1)\left(2^{k}|x-y|\right)^{\alpha+\beta_{1}-\delta}\left\|f_{1}\right\|_{L i p_{\alpha}\left(\mathbb{R}^{n}\right)} \\
& \lesssim\left\|f_{1}\right\|_{L i p_{\alpha}\left(\mathbb{R}^{n}\right)}\left\|f_{2}\right\|_{L^{n / \alpha}\left(\mathbb{R}^{n}\right)} .
\end{aligned}
$$

The estimates above yield, for any ball $Q$,

$$
\begin{aligned}
& \frac{1}{|Q|} \int_{Q}\left|\tilde{T}\left(f_{1}, f_{2}\right)(x)-m_{Q}\left[\tilde{T}\left(f_{1}, f_{2}\right)\right]\right| d x \\
& \quad=\frac{1}{|Q|} \int_{Q}\left|\frac{1}{|Q|} \int_{Q} \tilde{T}\left(f_{1}, f_{2}\right)(x)-\tilde{T}\left(f_{1}, f_{2}\right)(y) d y\right| d x \\
& \quad \lesssim \frac{1}{|Q|^{2}} \sum_{i=1}^{4} \int_{Q} \int_{Q}\left|\mathrm{I}_{\mathrm{i}}\right| d x d y \\
& \quad \lesssim\left\|f_{1}\right\|_{L i p_{\alpha}\left(\mathbb{R}^{n}\right)}\left\|f_{2}\right\|_{L^{n / \alpha}\left(\mathbb{R}^{n}\right)},
\end{aligned}
$$

that is, $\left\|\tilde{T}\left(f_{1}, f_{2}\right)\right\|_{B M O\left(\mathbb{R}^{n}\right)} \lesssim\left\|f_{1}\right\|_{L i p_{\alpha}\left(\mathbb{R}^{n}\right)}\left\|f_{2}\right\|_{L^{n / \alpha}\left(\mathbb{R}^{n}\right)}$.

(i) $\Rightarrow$ (ii). It follows from $\left\|\tilde{T}\left(1, f_{2}\right)\right\|_{B M O\left(\mathbb{R}^{n}\right)} \lesssim\|1\|_{L i p_{\alpha}\left(\mathbb{R}^{n}\right)}\left\|f_{2}\right\|_{L^{n / \alpha}\left(\mathbb{R}^{n}\right)}=0$ that

$$
\tilde{T}\left(1, f_{2}\right)(x)=\tilde{T}\left(1, f_{2}\right)\left(x_{0}\right)=0 .
$$

Thus the proof of Theorem 2.1 is completed.

Proof of Theorem 2.4 We only need to show that (ii) is sufficient. For simplicity, we may assume that $\left\|f_{1}\right\|_{L i p_{\alpha_{1}}\left(\mathbb{R}^{n}\right)}=\left\|f_{2}\right\|_{L i p_{\alpha_{2}}\left(\mathbb{R}^{n}\right)}=1$.

For any $x, y \in \mathbb{R}^{n}$, we have

$$
\tilde{T}\left(f_{1}, f_{2}\right)(x)-\tilde{T}\left(f_{1}, f_{2}\right)(y):=\mathrm{II}_{1}+\mathrm{II}_{2}+\mathrm{II}_{3}+\mathrm{II}_{4}+\mathrm{II}_{5},
$$

where

$$
\mathrm{II}_{1}=\lim _{\epsilon \rightarrow 0} \int_{\epsilon<\left|x-y_{2}\right|<3 r} \int_{\epsilon<\left|x-y_{1}\right|<3 r} K\left(x, y_{1}, y_{2}\right)\left(f_{1}\left(y_{1}\right)-f_{1}(x)\right)\left(f_{2}\left(y_{2}\right)-f_{2}(x)\right) d y_{1} d y_{2},
$$




$$
\begin{aligned}
\mathrm{II}_{2}= & -\lim _{\epsilon \rightarrow 0} \int_{\epsilon<\left|x-y_{2}\right|<3 r} \int_{\epsilon<\left|x-y_{1}\right|<3 r} K\left(y, y_{1}, y_{2}\right)\left(f_{1}\left(y_{1}\right)-f_{1}(x)\right)\left(f_{2}\left(y_{2}\right)-f_{2}(x)\right) d y_{1} d y_{2}, \\
\mathrm{II}_{3}= & \lim _{\epsilon \rightarrow 0} \int_{\epsilon<\left|x-y_{1}\right|<3 r} \int_{3 r \leq\left|x-y_{2}\right|}\left(K\left(x, y_{1}, y_{2}\right)-K\left(y, y_{1}, y_{2}\right)\right) \\
& \times\left(f_{1}\left(y_{1}\right)-f_{1}(x)\right)\left(f_{2}\left(y_{2}\right)-f_{2}(x)\right) d y_{1} d y_{2}, \\
\mathrm{II}_{4}= & \lim _{\epsilon \rightarrow 0} \int_{3 r \leq\left|x-y_{1}\right|} \int_{\epsilon<\left|x-y_{2}\right|<3 r}\left(K\left(x, y_{1}, y_{2}\right)-K\left(y, y_{1}, y_{2}\right)\right) \\
& \times\left(f_{1}\left(y_{1}\right)-f_{1}(x)\right)\left(f_{2}\left(y_{2}\right)-f_{2}(x)\right) d y_{1} d y_{2}, \\
\mathrm{II}_{5}= & \int_{3 r \leq\left|x-y_{1}\right|} \int_{3 r \leq\left|x-y_{2}\right|}\left(K\left(x, y_{1}, y_{2}\right)-K\left(y, y_{1}, y_{2}\right)\right)\left(f_{1}\left(y_{1}\right)-f_{1}(x)\right)\left(f_{2}\left(y_{2}\right)-f_{2}(x)\right) d y_{1} d y_{2},
\end{aligned}
$$

here $r=|x-y|$.

First we give the estimate for $\mathrm{II}_{1}$. For $f_{1} \in \operatorname{Lip}_{\alpha_{1}}\left(\mathbb{R}^{n}\right)$ and $f_{2} \in \operatorname{Lip}_{\alpha_{2}}\left(\mathbb{R}^{n}\right)$,

$$
\begin{aligned}
\left|\mathrm{II}_{1}\right| & \lesssim \int_{3 B} \int_{3 B} \frac{\left|x-y_{1}\right|^{\alpha_{1}}\left|x-y_{2}\right|^{\alpha_{2}}}{\left(\left|x-y_{1}\right|+\left|x-y_{2}\right|^{2 n}\right)} d y_{1} d y_{2} \\
& \lesssim \int_{3 B} \frac{1}{\left|x-y_{1}\right|^{n-\alpha_{1}}} d y_{1} \int_{3 B} \frac{1}{\left|x-y_{2}\right|^{n-\alpha_{2}}} d y_{2} \\
& \lesssim|x-y|^{\alpha_{1}+\alpha_{2}} .
\end{aligned}
$$

Now we give the decomposition of the term of $\mathrm{II}_{2}$,

$$
\begin{aligned}
-\mathrm{II}_{2}= & \lim _{\epsilon \rightarrow 0} \int_{\epsilon<\left|x-y_{2}\right|<3 r} \int_{\epsilon<\left|x-y_{1}\right|<3 r} K\left(y, y_{1}, y_{2}\right)\left(f_{1}\left(y_{1}\right)-f_{1}(y)\right)\left(f_{2}\left(y_{2}\right)-f_{2}(y)\right) d y_{1} d y_{2} \\
& +\lim _{\epsilon \rightarrow 0} \int_{\epsilon<\left|x-y_{2}\right|<3 r} \int_{\epsilon<\left|x-y_{1}\right|<3 r} K\left(y, y_{1}, y_{2}\right)\left(f_{1}(y)-f_{1}(x)\right)\left(f_{2}\left(y_{2}\right)-f_{2}(y)\right) d y_{1} d y_{2} \\
& +\lim _{\epsilon \rightarrow 0} \int_{\epsilon<\left|x-y_{2}\right|<3 r} \int_{\epsilon<\left|x-y_{1}\right|<3 r} K\left(y, y_{1}, y_{2}\right)\left(f_{1}\left(y_{1}\right)-f_{1}(y)\right)\left(f_{2}(y)-f_{2}(x)\right) d y_{1} d y_{2} \\
& +\lim _{\epsilon \rightarrow 0} \int_{\epsilon<\left|x-y_{2}\right|<3 r} \int_{\epsilon<\left|x-y_{1}\right|<3 r} K\left(y, y_{1}, y_{2}\right)\left(f_{1}(y)-f_{1}(x)\right)\left(f_{2}(y)-f_{2}(x)\right) d y_{1} d y_{2} \\
:= & \mathrm{II}_{21}+\mathrm{II}_{22}+\mathrm{II}_{23}+\mathrm{II}_{24} .
\end{aligned}
$$

For $B(x, 3 r) \subset B(y, 4 r)$, we have

$$
\left|\mathrm{II}_{21}\right| \lesssim|x-y|^{\alpha_{1}+\alpha_{2}}
$$

Choosing $0<\alpha_{21}, \alpha_{22}<\alpha_{2}$ with $\alpha_{2}=\alpha_{21}+\alpha_{22}$, then

$$
\begin{aligned}
\left|\mathrm{II}_{22}\right| & \lesssim|x-y|^{\alpha_{1}} \int_{B(y, 4 r)} \frac{1}{\left|y-y_{1}\right|^{n-\alpha_{21}}} d y_{1} \int_{B(y, 4 r)} \frac{1}{\left|y-y_{2}\right|^{n-\alpha_{22}}} d y_{2} \\
& \lesssim|x-y|^{\alpha_{1}+\alpha_{2}} .
\end{aligned}
$$

With a similar argument to $\mathrm{II}_{22}$, we obtain

$$
\left|\mathrm{II}_{23}\right| \lesssim|x-y|^{\alpha_{1}+\alpha_{2}}
$$


Since $\tilde{B}:=B(y, 2 r) \subset B(x, 3 r) \subset 2 \tilde{B}$, condition (2.3) and [10], Lemma 2, we have

$$
\begin{aligned}
\left|\mathrm{II}_{24}\right| \lesssim & \left|f_{1}(y)-f_{1}(x)\right|\left|f_{2}(y)-f_{2}(x)\right|\left|\lim _{\epsilon \rightarrow 0} \int_{\epsilon<\left|y-y_{2}\right|<2 r} \int_{\epsilon<\left|y-y_{1}\right|<2 r} K\left(y, y_{1}, y_{2}\right) d y_{1} d y_{2}\right| \\
& +\left|f_{1}(y)-f_{1}(x)\right|\left|f_{2}(y)-f_{2}(x)\right|\left|\lim _{\epsilon \rightarrow 0} \int_{\epsilon<\left|y-y_{2}\right|<2 r} \int_{3 B \backslash \tilde{B}} K\left(y, y_{1}, y_{2}\right) d y_{1} d y_{2}\right| \\
& +\left|f_{1}(y)-f_{1}(x)\right|\left|f_{2}(y)-f_{2}(x)\right|\left|\lim _{\epsilon \rightarrow 0} \int_{3 B \backslash \tilde{B}} \int_{\epsilon<\left|y-y_{1}\right|<2 r} K\left(y, y_{1}, y_{2}\right) d y_{1} d y_{2}\right| \\
& +\left|f_{1}(y)-f_{1}(x)\right|\left|f_{2}(y)-f_{2}(x)\right|\left|\int_{3 B \backslash \tilde{B}} \int_{3 B \backslash \tilde{B}} K\left(y, y_{1}, y_{2}\right) d y_{1} d y_{2}\right| \\
\lesssim & |x-y|^{\alpha_{1}+\alpha_{2}}\left|\lim _{\epsilon \rightarrow 0} \int_{\epsilon<\left|y-y_{2}\right|<2 r} \int_{\epsilon<\left|y-y_{1}\right|<2 r} K\left(y, y_{1}, y_{2}\right) d y_{1} d y_{2}\right| \\
& +|x-y|^{\alpha_{1}+\alpha_{2}}\left|\int_{B(x, 3 r) \backslash B(y, 2 r)} K_{\tilde{X}_{\tilde{B}}}\left(y, y_{1}\right) d y_{1}\right| \\
& +|x-y|^{\alpha_{1}+\alpha_{2}} \mid \int_{B(x, 3 r) \backslash B(y, 2 r)} K_{\chi_{\tilde{B}}\left(y, y_{2}\right) d y_{2} \mid} \frac{1}{\lesssim} \\
& +|x-y|^{\alpha_{1}+\alpha_{2}} \int_{2 \tilde{B} \backslash \tilde{B}} \int_{2 \tilde{B} \backslash \tilde{B}} \frac{1}{\left(\left|y-y_{1}\right|+\left|y-y_{2}\right|\right)^{2 n}} d y_{1} d y_{2} \\
\vdots & |y|^{\alpha_{1}+\alpha_{2}} .
\end{aligned}
$$

It is easy to verify that

$$
\left|\mathrm{II}_{\mathrm{i}}\right| \lesssim|x-y|^{\alpha_{1}+\alpha_{2}}, \quad i=3,4,5
$$

Therefore,

$$
\left|\tilde{T}\left(f_{1}, f_{2}\right)(x)-\tilde{T}\left(f_{1}, f_{2}\right)(y)\right| \lesssim|x-y|^{\alpha_{1}+\alpha_{2}}
$$

that is,

$$
\left\|\tilde{T}\left(f_{1}, f_{2}\right)\right\|_{L i p_{\alpha_{1}+\alpha_{2}}\left(\mathbb{R}^{n}\right)} \lesssim 1
$$

this completes the proof of Theorem 2.4.

Proof of Theorem 2.5 By $\|1\|_{L i p_{\alpha}\left(\mathbb{R}^{n}\right)}=\|1\|_{\dot{B}^{s}\left(\mathbb{R}^{n}\right)}=0$, it is clear that (i) $\Rightarrow$ (ii). Therefore, it suffices to prove that if $\tilde{T}\left(1, f_{2}\right)(x)=\tilde{T}\left(f_{1}, 1\right)(x)=\tilde{T}(1,1)(x) \equiv 0$, then

$$
\left\|\tilde{T}\left(f_{1}, f_{2}\right)\right\|_{\dot{B}^{s-\alpha}\left(\mathbb{R}^{n}\right)} \lesssim\left\|f_{1}\right\|_{L i p_{\alpha}\left(\mathbb{R}^{n}\right)}\left\|f_{2}\right\|_{\dot{B}^{s}\left(\mathbb{R}^{n}\right)} .
$$

It follows from $\tilde{T}\left(1, f_{2}\right)(x)=\tilde{T}\left(f_{1}, 1\right)(x)=\tilde{T}(1,1)(x) \equiv 0$ that, for any $x, y \in \mathbb{R}^{n}$,

$$
\begin{aligned}
& \tilde{T}\left(f_{1}, f_{2}\right)(x)-\tilde{T}\left(f_{1}, f_{2}\right)(y) \\
& =\lim _{\epsilon \rightarrow 0} \int_{\mathbb{R}^{n}} \int_{\mathbb{R}^{n}}\left(K_{\epsilon}\left(x, y_{1}, y_{2}\right)-K_{\epsilon}\left(y, y_{1}, y_{2}\right)\right) \\
& \quad \times\left(f_{1}\left(y_{1}\right)-f_{1}(x)\right)\left(f_{2}\left(y_{2}\right)-f_{2}(x)\right) \chi_{3 B}\left(y_{1}\right) \chi_{3 B}\left(y_{2}\right) d y_{1} d y_{2}
\end{aligned}
$$




$$
\begin{aligned}
= & \lim _{\epsilon \rightarrow 0} \int_{\mathbb{R}^{n}} \int_{\mathbb{R}^{n}} K_{\epsilon}\left(x, y_{1}, y_{2}\right)\left(f_{1}\left(y_{1}\right)-f_{1}(x)\right)\left(f_{2}\left(y_{2}\right)-f_{2}(x)\right) \chi_{3 B}\left(y_{1}\right) \chi_{3 B}\left(y_{2}\right) d y_{1} d y_{2} \\
& -\lim _{\epsilon \rightarrow 0} \int_{\mathbb{R}^{n}} \int_{\mathbb{R}^{n}} K_{\epsilon}\left(y, y_{1}, y_{2}\right)\left(f_{1}\left(y_{1}\right)-f_{1}(x)\right)\left(f_{2}\left(y_{2}\right)-f_{2}(x)\right) \chi_{3 B}\left(y_{1}\right) \chi_{3 B}\left(y_{2}\right) d y_{1} d y_{2} \\
& +\lim _{\epsilon \rightarrow 0} \int_{\mathbb{R}^{n}} \int_{\mathbb{R}^{n}}\left(K_{\epsilon}\left(x, y_{1}, y_{2}\right)-K_{\epsilon}\left(y, y_{1}, y_{2}\right)\right) \\
& \times\left(f_{1}\left(y_{1}\right)-f_{1}(x)\right)\left(f_{2}\left(y_{2}\right)-f_{2}(x)\right) \chi_{(3 B)^{c}}\left(y_{1}\right) \chi_{3 B}\left(y_{2}\right) d y_{1} d y_{2} \\
& +\lim _{\epsilon \rightarrow 0} \int_{\mathbb{R}^{n}} \int_{\mathbb{R}^{n}}\left(K_{\epsilon}\left(x, y_{1}, y_{2}\right)-K_{\epsilon}\left(y, y_{1}, y_{2}\right)\right) \\
& \times\left(f_{1}\left(y_{1}\right)-f_{1}(x)\right)\left(f_{2}\left(y_{2}\right)-f_{2}(x)\right) \chi_{3 B}\left(y_{1}\right) \chi_{(3 B)} c\left(y_{2}\right) d y_{1} d y_{2} \\
& +\lim _{\epsilon \rightarrow 0} \int_{\mathbb{R}^{n}} \int_{\mathbb{R}^{n}}\left(K_{\epsilon}\left(x, y_{1}, y_{2}\right)-K_{\epsilon}\left(y, y_{1}, y_{2}\right)\right) \\
& \times\left(f_{1}\left(y_{1}\right)-f_{1}(x)\right)\left(f_{2}\left(y_{2}\right)-f_{2}(x)\right) \chi_{(3 B)}\left(y_{1}\right) \chi_{(3 B)}\left(y_{2}\right) d y_{1} d y_{2} \\
:= & \mathrm{III}_{1}+\mathrm{II}_{2}+\mathrm{III}_{3}+\mathrm{III}_{4}+\mathrm{III}_{5},
\end{aligned}
$$

where $B=B(x,|x-y|)$.

For $\mathrm{III}_{1}$, we choose $\gamma$ such that $0<\gamma<s-\alpha$, by $\left|x-y_{2}\right|^{-n}=\left|x-y_{2}\right|^{-n / 2-\gamma}\left|x-y_{2}\right|^{-n / 2+\gamma}$ and the Hölder inequality, we have

$$
\begin{aligned}
\left|\mathrm{II}_{1}\right| & \lesssim\left\|f_{1}\right\|_{L i p_{\alpha}\left(\mathbb{R}^{n}\right)} \int_{3 B} \int_{3 B} \frac{\left|f_{2}\left(y_{2}\right)-f_{2}(x)\right|\left|x-y_{1}\right|^{\alpha}}{\left(\left|x-y_{1}\right|+\left|x-y_{2}\right|\right)^{2 n}} d y_{1} d y_{2} \\
& \lesssim\left\|f_{1}\right\|_{L i p_{\alpha}\left(\mathbb{R}^{n}\right)} \int_{3 B} \frac{1}{\left|x-y_{1}\right|^{n-\alpha}} d y_{1} \int_{3 B} \frac{\left|f_{2}\left(y_{2}\right)-f_{2}(x)\right|}{\left|x-y_{2}\right|^{n}} d y_{2} \\
& \lesssim\left\|f_{1}\right\|_{L i p_{\alpha}\left(\mathbb{R}^{n}\right)|x-y|^{\alpha}}\left(\int_{3 B} \frac{\left|f_{2}\left(y_{2}\right)-f_{2}(x)\right|^{2}}{\left|x-y_{2}\right|^{n+2 \gamma}} d y_{2}\right)^{1 / 2}\left(\int_{3 B} \frac{1}{\left|x-y_{2}\right|^{n-2 \gamma}} d y_{2}\right)^{1 / 2} \\
& \lesssim\left\|f_{1}\right\|_{L i p_{\alpha}\left(\mathbb{R}^{n}\right)|x-y|^{\gamma+\alpha}}\left(\int_{3 B} \frac{\left|f_{2}\left(y_{2}\right)-f_{2}(x)\right|^{2}}{\left|x-y_{2}\right|^{n+2 \gamma}} d y_{2}\right)^{1 / 2},
\end{aligned}
$$

then

$$
\begin{aligned}
\int_{\mathbb{R}^{n}} \int_{\mathbb{R}^{n}} \frac{\left|I I I_{1}\right|^{2}}{|x-y|^{n+2 s}} d x d y & \lesssim\left\|f_{1}\right\|_{L i p_{\alpha}\left(\mathbb{R}^{n}\right)}^{2} \int_{\mathbb{R}^{n}} \int_{\mathbb{R}^{n}} \frac{\left|f_{2}\left(y_{2}\right)-f_{2}(x)\right|^{2}}{\left|x-y_{2}\right|^{n+2 s-2 \alpha}} d x d y_{2} \\
& \lesssim\left\|f_{1}\right\|_{L i p_{\alpha}\left(\mathbb{R}^{n}\right)}^{2}\left\|f_{2}\right\|_{\dot{B}^{s-\alpha}\left(\mathbb{R}^{n}\right)^{\prime}}^{2}
\end{aligned}
$$

Next, we give the estimate for $\mathrm{III}_{2}$. Observe that $\tilde{B}:=B(y, 2|x-y|) \subset B(x, 3|x-y|) \subset 2 \tilde{B}$ and

$$
\begin{aligned}
\left|\mathrm{III}_{2}\right| \lesssim & \left|\int_{3 B} \int_{3 B} K\left(y, y_{1}, y_{2}\right)\left(f_{1}\left(y_{1}\right)-f_{1}(y)\right)\left(f_{2}\left(y_{2}\right)-f_{2}(y)\right) d y_{1} d y_{2}\right| \\
& +\left|\int_{3 B} \int_{3 B} K\left(y, y_{1}, y_{2}\right)\left(f_{1}\left(y_{1}\right)-f_{1}(y)\right)\left(f_{2}(y)-f_{2}(x)\right) d y_{1} d y_{2}\right| \\
& +\left|\int_{3 B} \int_{3 B} K\left(y, y_{1}, y_{2}\right)\left(f_{1}(y)-f_{1}(x)\right)\left(f_{2}\left(y_{2}\right)-f_{2}(y)\right) d y_{1} d y_{2}\right| \\
& +\lim _{\epsilon \rightarrow 0}\left|\int_{\tilde{B}} \int_{\tilde{B}} K_{\epsilon}\left(y, y_{1}, y_{2}\right)\left(f_{1}(y)-f_{1}(x)\right)\left(f_{2}(y)-f_{2}(x)\right) d y_{1} d y_{2}\right|
\end{aligned}
$$




$$
\begin{aligned}
& \quad+\lim _{\epsilon \rightarrow 0}\left|\int_{\tilde{B}} \int_{3 B \backslash \tilde{B}} K_{\epsilon}\left(y, y_{1}, y_{2}\right)\left(f_{1}(y)-f_{1}(x)\right)\left(f_{2}(y)-f_{2}(x)\right) d y_{1} d y_{2}\right| \\
& \quad+\lim _{\epsilon \rightarrow 0}\left|\int_{3 B \backslash \tilde{B}} \int_{\tilde{B}} K_{\epsilon}\left(y, y_{1}, y_{2}\right)\left(f_{1}(y)-f_{1}(x)\right)\left(f_{2}(y)-f_{2}(x)\right) d y_{1} d y_{2}\right| \\
& \quad+\lim _{\epsilon \rightarrow 0}\left|\int_{3 B \backslash \tilde{B}} \int_{3 B \backslash \tilde{B}} K_{\epsilon}\left(y, y_{1}, y_{2}\right)\left(f_{1}(y)-f_{1}(x)\right)\left(f_{2}(y)-f_{2}(x)\right) d y_{1} d y_{2}\right| \\
& :=\mathrm{III}_{21}+\mathrm{III}_{22}+\mathrm{III}_{23}+\mathrm{III}_{24}+\mathrm{III}_{25}+\mathrm{III}_{26}+\mathrm{III}_{27} .
\end{aligned}
$$

With a similar argument to the estimate for $\mathrm{III}_{1}$, we obtain

$$
\int_{\mathbb{R}^{n}} \int_{\mathbb{R}^{n}} \frac{\left|\mathrm{III}_{2 \mathrm{j}}\right|^{2}}{|x-y|^{n+2 s}} d x d y \lesssim\left\|f_{1}\right\|_{L i p_{\alpha}\left(\mathbb{R}^{n}\right)}^{2}\left\|f_{2}\right\|_{\dot{B}^{s-\alpha}\left(\mathbb{R}^{n}\right)}^{2}, \quad j=1,3 .
$$

For $\mathrm{III}_{22}$, choose $0<\alpha_{1}, \alpha_{2}<\alpha$ such that $\alpha_{1}+\alpha_{2}=\alpha$, then

$$
\begin{aligned}
\left|I I_{22}\right| & \lesssim\left\|f_{1}\right\|_{L i p_{\alpha}\left(\mathbb{R}^{n}\right)}\left|f_{2}(y)-f_{2}(x)\right| \int_{\tilde{B}} \int_{\tilde{B}} \frac{\left|y-y_{1}\right|^{\alpha}}{\left(\left|y-y_{1}\right|+\left|y-y_{2}\right|\right)^{2 n}} d y_{1} d y_{2} \\
& \lesssim\left\|f_{1}\right\|_{L i p_{\alpha}\left(\mathbb{R}^{n}\right)}\left|f_{2}(y)-f_{2}(x)\right| \int_{\tilde{B}} \frac{1}{\left|y-y_{1}\right|^{n-\alpha_{1}}} d y_{1} \int_{\tilde{B}} \frac{1}{\left|y-y_{2}\right|^{n-\alpha_{2}}} d y_{2} \\
& \lesssim\left\|f_{1}\right\|_{L i p_{\alpha}\left(\mathbb{R}^{n}\right)}|x-y|^{\alpha}\left|f_{2}(y)-f_{2}(x)\right| .
\end{aligned}
$$

To control $\mathrm{III}_{24}$ we use condition (2.3), that is, for any $0<r<R$,

$$
\left|\int_{r<\left|x-y_{1}\right|<R} \int_{r<\left|x-y_{2}\right|<R} K\left(x, y_{1}, y_{2}\right) d y_{1} d y_{2}\right| \leq C
$$

to obtain

$$
\int_{\mathbb{R}^{n}} \int_{\mathbb{R}^{n}} \frac{\left|\mathrm{III}_{24}\right|^{2}}{|x-y|^{n+2 s}} d x d y \lesssim\left\|f_{1}\right\|_{L i p_{\alpha}\left(\mathbb{R}^{n}\right)}^{2}\left\|f_{2}\right\|_{\dot{B}^{s-\alpha}\left(\mathbb{R}^{n}\right)}^{2}
$$

The argument for $\mathrm{III}_{25}$ and $\mathrm{III}_{26}$ is similar, we only give the estimate for $\mathrm{III}_{25}$. By Remark 2.1 and $3 B \subset 2 \tilde{B}$, we obtain

$$
\begin{aligned}
\left|\mathrm{II}_{25}\right| & \lesssim|x-y|^{\alpha}\left|f_{2}(y)-f_{2}(x)\right|\left|\int_{3 B \backslash \tilde{B}} \int_{\mathbb{R}^{n}} K\left(y, y_{1}, y_{2}\right) \chi_{\tilde{B}}\left(y_{2}\right) d y_{1} d y_{2}\right| \\
& \lesssim|x-y|^{\alpha}\left|f_{2}(y)-f_{2}(x)\right|\left|\int_{3 B \backslash \tilde{B}} K_{\chi_{\tilde{B}}}\left(y, y_{1}\right) d y_{1}\right| \\
& \lesssim|x-y|^{\alpha}\left|f_{2}(y)-f_{2}(x)\right| \int_{\tilde{2} \tilde{B} \mid \tilde{B}} \frac{1}{\left|y-y_{1}\right|^{n}} d y_{1} \\
& \lesssim|x-y|^{\alpha}\left|f_{2}(y)-f_{2}(x)\right| .
\end{aligned}
$$

Finally, it is easy to see that

$$
\begin{aligned}
\left|\mathrm{III}_{27}\right| & \lesssim|x-y|^{\alpha}\left|f_{2}(y)-f_{2}(x)\right| \int_{2 \tilde{B} \backslash \tilde{B}} \int_{2 \tilde{B} \backslash \tilde{B}} \frac{1}{\left(\left|y-y_{1}\right|+\left|y-y_{2}\right|\right)^{2 n}} d y_{1} d y_{2} \\
& \lesssim|x-y|^{\alpha}\left|f_{2}(y)-f_{2}(x)\right| .
\end{aligned}
$$


We only give the estimate for $\mathrm{III}_{3}$. For the estimate for $\mathrm{III}_{4}$ and $\mathrm{III}_{5}$ similar arguments are applied with necessary modifications. For $\mathrm{III}_{3}$, since $\left|K\left(x, y_{1}, y_{2}\right)-K\left(y, y_{1}, y_{2}\right)\right| \lesssim$ $\frac{|x-y|^{\delta}}{\left(\left|x-y_{1}\right|+\left|x-y_{2}\right|\right)^{2 n+\delta}}$,

$$
\begin{aligned}
\left|I I I_{3}\right| & \lesssim\left\|f_{1}\right\|_{L i p_{\alpha}\left(\mathbb{R}^{n}\right)} \int_{2 B} \int_{\mathbb{R}^{n} \backslash 2 B} \frac{|x-y|^{\delta}\left|f_{1}\left(y_{1}\right)-f_{1}(x)\right|\left|x-y_{2}\right|^{\alpha}}{\left(\left|x-y_{1}\right|+\left|x-y_{2}\right|\right)^{2 n+\delta}} d y_{1} d y_{2} \\
& \lesssim\left\|f_{1}\right\|_{L i p_{\alpha}\left(\mathbb{R}^{n}\right)}|x-y|^{\delta} \int_{2 B} \frac{\left|f_{1}\left(y_{1}\right)-f_{1}(x)\right|}{\left|x-y_{1}\right|^{n}} d y_{1} \int_{\mathbb{R}^{n} \backslash 2 B}\left|x-y_{2}\right|^{-n-\delta+\alpha} d y_{2} \\
& \lesssim\left\|f_{1}\right\|_{L i p_{\alpha}\left(\mathbb{R}^{n}\right)|x-y|^{\alpha}} \int_{2 B} \frac{\left|f_{1}\left(y_{1}\right)-f_{1}(x)\right|}{\left|x-y_{1}\right|^{n}} d y_{1} \\
& \lesssim\left\|f_{1}\right\|_{L i p_{\alpha}\left(\mathbb{R}^{n}\right)}|x-y|^{\alpha+\gamma}\left(\int_{|y-x| \leq 2\left|x-y_{1}\right|} \frac{\left|f_{1}\left(y_{1}\right)-f_{1}(x)\right|^{2}}{\left|x-y_{1}\right|^{n+2 \gamma}} d y_{1}\right)^{1 / 2} .
\end{aligned}
$$

Thus, $\int_{\mathbb{R}^{n}} \int_{\mathbb{R}^{n}} \frac{\left|I I I_{3}\right|^{2}}{\left.|x-y|\right|^{n+2 s}} d x d y \lesssim\left\|f_{1}\right\|_{L i p_{\alpha}\left(\mathbb{R}^{n}\right)}^{2}\left\|f_{2}\right\|_{\dot{B}^{s-\alpha}\left(\mathbb{R}^{n}\right)^{2}}^{2}$.

Combining the estimates above, we obtain

$$
\int_{\mathbb{R}^{n}} \int_{\mathbb{R}^{n}} \frac{\left|\tilde{T}\left(f_{1}, f_{2}\right)(x)-\tilde{T}\left(f_{1}, f_{2}\right)(y)\right|^{2}}{|x-y|^{n+2 s}} d x d y \lesssim\left\|f_{1}\right\|_{L i p_{\alpha}\left(\mathbb{R}^{n}\right)}^{2}\left\|f_{2}\right\|_{\dot{B}^{s-\alpha}\left(\mathbb{R}^{n}\right)}^{2}
$$

This concludes the proof of the theorem.

\section{Competing interests}

The authors declare that they do not have any commercial or associative interest that represents a conflict of interest in connection with the work submitted.

\section{Authors' contributions}

All authors contributed equally to the writing of this paper. All authors read and approved the final manuscript.

\section{Acknowledgements}

The research was supported by Xinjiang Graduate Research and Innovation Project (Grant No. 2014011) and National Natural Science Foundation of China (Grant No. 11261055).

Received: 19 April 2015 Accepted: 29 November 2015 Published online: 09 December 2015

\section{References}

1. Coifman, R, Rochberg, R, Weiss, G: Factorization theorems for Hardy spaces in several variables. Ann. Math. 103, 611-635 (1976)

2. Lemarié, PG: Continuité sur les espaces de Besov des opérateurs définis par des intégrales singulières. Ann. Inst. Fourier (Grenoble) 35, 175-187 (1985)

3. Meyer, Y: Continuité sur les espaces de Holder et de Sobolev des opérateurs définis par des intégrales singulières. In: Peral, I, Rubio de Francia, J-L (eds.) Recent Progress in Fourier Analysis, pp. 145-172 (1985)

4. Meyer, Y, Cofiman, R: Wavelets: Caldrón-Zygmund and Multilinear Operators. Cambridge Studies in Advanced Mathematics, vol. 48. Cambridge University Press, Cambridge (1997)

5. Gatto, AE, García-Cuerva, J: Lipschitz spaces and Calderón-Zygmund operators associated to non-doubling measures. Publ. Math. 49, 285-296 (2005)

6. Zhou, J, Wang, DH: Lipschitz spaces and fractional integral operators associated to non-homogeneous metric measure spaces. Abstr. Appl. Anal. 2014, 174010 (2014)

7. Kenig, C, Stein, E: Multilinear estimates and fractional integration. Math. Res. Lett. 6, 1-15 (1999)

8. Coifman, RR, Meyer, Y: On commutators of singular integral and bilinear singular integrals. Trans. Am. Math. Soc. 212, 315-331 (1975)

9. Coifman, RR, Meyer, Y: Commutateurs d'intégrales singulières et opérateurs multilinéaires. Ann. Inst. Fourier (Grenoble) 28, 177-202 (1978)

10. Grafakos, L, Torres, R: Multilinear Calderón-Zygmund theory. Adv. Math. 165, 124-164 (2002)

11. Grafakos, L, Kalton, N: Multilinear Calderón-Zygmund operators on Hardy spaces. Collect. Math. 52, 169-179 (2001)

12. Grafakos, L, Torres, R: On multilinear singular integrals of Calderón-Zygmund type. Publ. Math. 46, Extra, 57-91 (2000)

13. Grafakos, L, Torres, R: Maximal operator and weighted norm inequalities for multilinear singular integrals. Indiana Univ. Math. J. 51, 1261-1276 (2002)

14. Hörmander, L: The Analysis of Linear Partial Differential Operators I, 2nd edn. Springer, Berlin (1990)

15. Benedek, A, Calerón, AP, Panzone, R: Convolution operators on Banach space valued functions. Proc. Natl. Acad. Sci. USA 48, 356-365 (1962) 